\title{
The impact of metallicity on the formation of pre-collapsing minihalos
}

\author{
Aycin Aykutalp ${ }^{1}$ and Marco Spaans ${ }^{1}$ \\ ${ }^{1}$ Kapteyn Astronomical Institute, University of Groningen, \\ Postbus 800, 9700 AV, Groningen, the Netherlands \\ email: aykutalp@astro.rug.nl, spaans@astro.rug.nl
}

\begin{abstract}
In this study we consider the pre-enrichment of minihalos, and study the impact of metallicity on pre-collapsing minihalos by using the cosmological, N-body simulation code Enzo. The metallicities that we consider are assumed to be the result of pre-enrichment by earlier star formation. In the simulations of $10^{-3}$ and $10^{-1} \mathrm{Z}_{\odot}$ we see a big difference for the collapse of the minihalo. In the high metallicity case the minihalo is more compact compared to the low metallicity case and we reach higher densities due to the efficient cooling. Also in the high metallicity case the gas cools down to lower temperatures and we see cold, dense gas which indicates a multi-phase ISM. This leads us to think that there is a transition region between metallicities of $10^{-3}$ and $10^{-1} \mathrm{Z}_{\odot}$ which lowers the mass scale of the next generation of stars. Furthermore, because the gas cools more efficiently in the high metallicity case there is less pressure support against gravity and therefore we see higher velocities.
\end{abstract}

\section{Introduction}

In the last two decades, cosmological simulations have become an important tool for theoreticians to simulate the formation of structure from primordial fluctuations. According to the CDM model of hierarchical structure formation, the first (PopIII) stars have formed at redshifts of $\mathrm{z}=20-30$, in dark matter halos of $10^{6} \mathrm{M}_{\odot}$ (Tegmark et al. 1997). The formation of a star depends on the ability of interstellar gas to cool and form dense molecular gas. Therefore chemical composition and metallicity of the interstellar gas are the key parameters to study. To investigate the effect of metallicity on the transition from a top-heavy IMF to the current IMF we have run simulations of pre-collapsing minihalos with different metallicities by using the adaptive mesh refinement code, Enzo (Bryan \& Norman 1997).

In our simulation we use $128^{3}$ grid cells on the top grid with three nested subgrids, each refining by a factor of two. The box size of the simulation is $0.25 \mathrm{~h}^{-1} \mathrm{Mpc}$. The resolution of our simulations is $0.1 \mathrm{pc}$ at redshift 15 . We run simulations from redshift 99 to 5 and use three different cooling prescriptions for metallicities of $10^{-3}, 10^{-2}, 10^{-1} \mathrm{Z}_{\odot}$. In these simulations, we ignore the UV background radiation and feedback effects from the PopIII stars. The metallicity dependent cooling includes finestructure emissions from carbon and oxygen as well as molecular lines from species like CO. All level populations are computed under statistical equilibrium. The chemistry includes gas phase and grain surface formation of $\mathrm{H}_{2}$ and $\mathrm{HD}$.

In the near future, in order to understand the nature of the first stars and their feedback effects we are going to implement the PDR and XDR codes of Spaans \& Meijerink (2008) with updated grain surface formation of $\mathrm{H}_{2}$ and HD from Cazaux \& Spaans (2009) into Enzo. 


\section{Results}

Figure 1 shows the density-temperature profile for a minihalo for metallicities of $10^{-3}$ and $10^{-1} \mathrm{Z}_{\odot}$ at $\mathrm{z}=15$. In the high metallicity case we see cold dense gas which indicates a multiphase ISM while we lack this gas in the low metallicity case. This is because in the low metallicity case the gas cannot cool efficiently enough to lower temperatures. Also, due to the shorter cooling time scale we see that in the high metallicity case the minihalo evolves faster dynamically. This can be seen in figure 2 which shows the magnitudes of the total velocities in a slice of the simulation. Because the gas cools more efficiently there is less pressure support against gravity and therefore the high metallicity case reaches higher velocities.
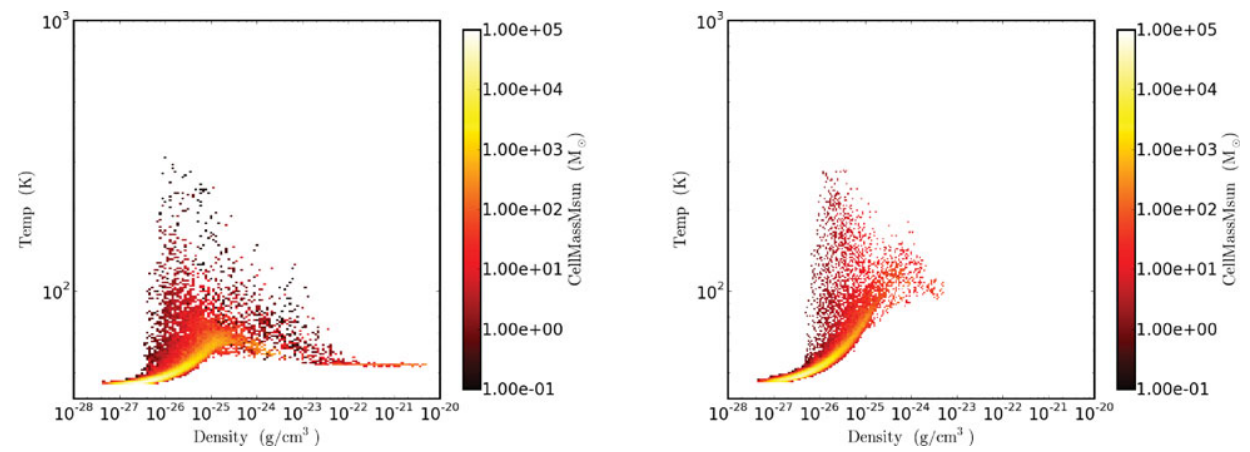

Figure 1. Density-temperature profile of the central $2 \mathrm{kpc}$ of a minihalo at redshift 16 for metallicities of $10^{-1}$ (left) and $10^{-3}$ (right) $\mathrm{Z}_{\odot}$. The color scheme indicates the distribution of each bin.
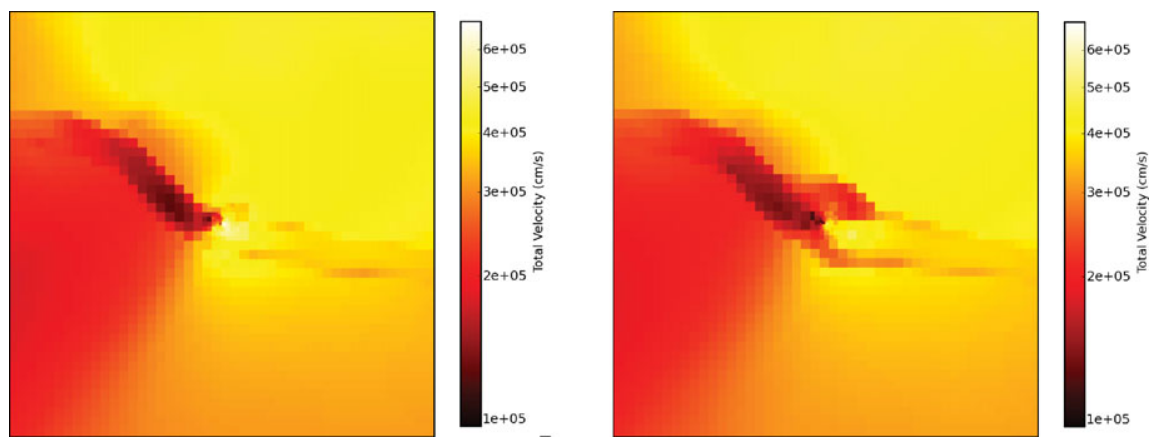

Figure 2. Magnitudes of the total velocities of a minihalo for the central $2 \mathrm{kpc}$ for metallicities of $10^{-1}$ (left) and $10^{-3}$ (right) $\mathrm{Z}_{\odot}$ at redshift 15 .

\section{References}

Bryan, G. \& Norman, M. 1997, ASPC, 123, 363B

Cazaux, S. \& Spaans, M. 2009, $A \& A$, 496, 365C

Spaans, M. \& Meijerink, R. 2008, ApJ, 678L, 5

Tegmark, M., Silk, J., Rees, M. J., Blanchard, A., Abel, T., \& Palla, F. 1997, ApJ, 474, 1 